to be sited in small towns or villages and should be encouraged. No modern industry has been suggested which would provide seasonal or part-time employment for agricultural workers and their families, and appropriate bodies should be asked to make a special study of this problem. Before new towns are established in country areas, vacant or derelict industrial sites in existing towns should be fully utilized. New satellite towns, housing estates, garden cities and suburbs should be sited wherever practicable away from the better farm land, and due attention paid to agricultural considerations in their siting. Sporadie buildings in villages and rural areas should be controlled, and new villages and extensions of villages should be planned and should be so far as possible of a compact and closely knit character. All new villages and country towns should be situated away from main traffic roads though within easy access of them, and all existing villages and country towns which have main traffic roads running through them should be by-passed so far as possible. New buildings in country areas should use to the full all the possibilities which new materials and new building tech. niques have made available, though the buildings should be designed so as to harmonize with their surroundings.

\section{THEORY OF X-RAY DIFFRACTION}

\section{By Prof. P. P. EWALD}

Queen's University, Belfast

$\mathrm{T}$ $\mathrm{HE}$ numerous books on the diffraction of X-rays by crystals all treat the subject with the view of introducing the reader to the methods for determining erystal structures. It is true that some presentation of the theory of optical interference cannot be omitted and that the various books go into this theory to varying degrees. But it is only v. Laue's recent book* which makes the optical theory of X-ray interference in crystals its main aim. In it, the usual methods of crystal structure determination are curtailed or omitted, since they involve more geometrical than optical discussion, once the reduced intensities are taken as known. Omitted as well is the description of experimental methods, as the emphasis of the book is on theory.

What variety and scope the subject presents will become apparent on comparing it with the corresponding theory of the diffraction of light by a plane optical grating. This theory consists of two parts : the diffraction by a single element of the grating (slit, wire or groove) and the action produced by the repetition of such elements. The first part is not important for most aspects of the effect of a plane grating, other than the distribution of intensities over the spectra of different orders. Its counterpart in X-ray diffraction is the theory of the atomic factor, and this brings the subject at once into the domain of wavemechanical atomic theory.

The second part of the theory, the grating effect proper, is more involved for X-rays by the fact that they encounter in a crystal a three-dimensional grating. This fact leads to geometrical propositions concerning the main directions of diffraction which equal in beauty the geometrical constructions of the Fresnel-Hamiltonian crystal optics. Furthermore, the

* Röntgenstrahlinterferenzen. Von Prof. M. v. Laue. Pp. viii+358. (Leipzig : Akademische Verlagsgesellschaft Becker und Erler Kom.
Ges., 1941.) three-dimensionality of the crystal shape is carried over to the array of secondary diffraction maxima which surround each main maximum. The resulting complicated intensity distribution in space, modified by the 'erystal shape factor', is the three-dimensional generalization of the well-known one-dimensional diffraction function which determines the resolving power of a plane grating of finite width.

The most profound influence of the threedimensionality is, however, to be found in the strong interaction of each element with all its neighbours; this makes the theory of diffraction a part of the major problem of the propagation of an optical field through the erystal, that is, part of the problem of refraction and dispersion by the crystal. The resulting 'dynamical theory' of X-ray diffraction is fully treated in Laue's book.

While these points still bear a correspondence to the problems encountered for the plane grating, there are three others which have no counterpart. The first of these is due to the thermal motion of the diffracting centres, which not only produces an unavoidable irregularity of the lattice but also endows each centre with a velocity leading to a Doppler effect of the scattered radiation. The second new point is the production of scattered radiation of incoherent character and of Compton-modified frequency. The last is connected with the possibility of generating, for example by electronic bombardment of the crystal, monochromatic radiation at the interior of the crystal; this radiation has been shown by Kossel to emerge preferentially in certain directions.

The aspects of the theory as enumerated determine the scheme of Laue's book. The author starts with the theory of atomic scattering along wave-mechanical lines, but in rather a novel manner. He first points out that the general theory of integration of Maxwell's equations permits the introduction of various forms of scalar and vector potentials. Now for the perturbation problem of finding the scattered radiation without blurring the distinction between the perturbing incident potential and the normal constitutional potential of the atom, only one of these forms is suitable, namely that in which the fields derived from the vector potential cannot cancel those derived from the scalar potential. This is true for the atom as well as for the entire crystal, and the correct introduction of the potential is essential for the unity of method in treating atoms and crystals. The full development of the method will be found in the paragraphs introducing the dynamical theory, where the wave-mechanical concepts are further related to classical Maxwellian theory. The existence of the Schroedinger current can be interpreted classically as due to a changing polarization, and the polarization together with the electric field vector $E$ can be compounded to a dielectric displacement $D$ This is as in Lorentz's theory of electrons, except for the difference that $D$ is here a vector locally determined by the $\psi$-function for each electron, and not an average value over a distribution of polarization unknown in detail. The distinction between $E$ and $D$ leads to the definition of a dielectric constant which for a crystal is a periodic function in space. The theory of diffraction and of scattering is thus reduced to the problem of integrating the Maxwellian equations for the propagation of waves in a medium of varying dielectric constant. These variations are determined, according to wave-mechanics, by the ground state of the system as long as absorption is negligible, and by higher states as well near absorption edges. 
The reduction of the problem of X-ray diffraction to wave-mechanics has been developed in papers by Laue and his co-workers Kohler, Moliere, Lamla, but it is given a more connected discussion in this book. Its main advantage is the uniformity of method in treating the scattering by atoms, molecules and crystals, and for the latter the foundation of the dynamical theory on the Fourier series for the electron density in the crystal.

Among the problems that have to be dealt with by the dynamical theory are the intensity of reflexion by a perfect crystal, the multiple reflexion resulting in a weakening (Aufhellung) or strengthening (Umweganregung) of the main reflexion, and the "lattice source method" of diffraction of Kossel. The latter has been reduced in a most elegant way by Laue to the established formalism of the dynamical theory by using an optical reciprocity theorem which relates the intensity received outside the crystal from a source inside it to the intensity created by an external source at the point of emission within the crystal.

Apart from the dynamical theory, the optical side of the theory of the temperature factor has been treated with great care and clarity by v. Laue. It is true that this theory remains on the level of the 'kinematical' theory of diffraction which includes only such effects as result from composing scattered wavelets derived from a single primary (incident) wave of given propagation. Instead, the treatment to be aimed at should be to study the propagation of an optical field, consisting of an indefinite number of waves of adaptable velocities, through the temperature-disturbed erystal, in the same way as it is done for the undisturbed crystal in the dynamical theory.

This combined thermal-dynamical theory has so far not been attempted. Laue's treatment is, however, superior to most previous presentations in employing a uniform method for discussing the temperature effect on the scattering by molecules and by crystals. In both systems the thermal displacements can be regarded as due to superimposed normal oscillations about the equilibrium position, and the independent co-ordinates of the system are its normal co-ordinates which perform simple harmonic oscillations of frequencies, say, $v_{j}$. As a result of these, the scattered radiation can be expressed as a sum of components of Doppler-modified frequencies $\omega \pm p v_{j}$, where $\omega$ is the X-ray frequency, and $p$ any integer. The intensities of the components are given by Bessel functions, the arguments of which are the 'roughnesses' produced in the reflecting plane by each type of lattice vibration. The main difficulty of the calculation lies in the fact that since the 'roughness' is of the order of the X-ray wave-length, the change of phase produced by the superposition of lattice vibrations is not simply the sum of the phase differences due to each vibration. The result of Laue's careful discussion is that wavelets of unmodified frequency $(p=0)$ are collected in principal and secondary maxima of the same directions as for a lattice without thermal motion; the atomic factors $f_{n}$ have to be replaced by $f_{n} D_{n}$, where $D_{n}$ is a Debye factor containing in its argument the mean square displacement of the $n$th kind of atom taken along the normal to the reflecting plane. All the background scattering is due to radiation of Doppler-modified frequencies $\omega \pm p v_{j}$ with $p \neq 0$, but the change of frequency is too small to be readily detected by experiment. In stressing the Doppler effect, Laue's theory of the thermal factor differs from the recent treatment on wave-mechanical lines given by Born and Miss Sarginson and others, but it seems unlikely that the results would be greatly affected.

As a second step, of purely dynamical, not optical, theory, the mean square displacements of the atoms, resolved along the normal to the reflecting planes, have to be calculated as functions of the temperature. Laue's book, stressing the optical part, does no more than outline this problem.

In the last third of the book, which deals with the dynamical theory, many interesting details will be found apart from the general approach which has already been described above. The theory of Renninger's 'multiple reflection' and of Kossel's 'crystal source method' are worked out; with respect to the former it seems a pity that no attention has been given to the experimental and theoretical work of Weigle and co-workers. A careful discussion is given of the transition from the dynamical to the kinematical theory in the case of small crystals. Another item is the dynamical theory for frequencies near to an absorption edge, following a paper by G. Moliere.

Anyone familiar with the subject will appreciate the sound physical and mathematical basis of v. Laue's book, and the many original and critical ideas incorporated in it. The reading is not difficult in spite of the fairly involved mathematics, and the limitation of the book to points of major optical interest excludes detail which may be necessary for the discussion of experimental data, but which would fatigue the general reader. It is to be hoped that the author may find it possible to complete a similar account of the theory of electron diffraction, a closely related subject on which he has been engaged for the last ten years simultaneously with the subject presented in this volume.

\section{KEY METALS AND THE LOCATION OF INDUSTRY*}

\author{
BY Prof. C. B. FAWCETT \\ University College, London
}

$\mathrm{F}$ ROM the beginnings of man's knowledge of metals up to about two hundred years ago, the number of known metals could be counted on his fingers. There were the 'noble', but not particularly useful, gold and silver, and such 'base' metals as copper, iron and lead, which made up for their baseness by being useful. Mercury also has long been known. Bronze and brass were known; though it is doubtful whether their composition and their relations to copper, tin, and zine and antimony were understood before the eighteenth and nineteenth centuries. The number now known is five times as great. But, except for aluminium, the newer metals are chiefly of importance for their part in the formation of many alloys with the older metals. Iron is still by far the most important base of such alloys, and the most important of all the metals.

The effects on human society of so great an addition to available natural resources are both complex and far-reaching. But here I wish to note only some effects of the use of the key metals on the location

* Paper read at the Conference on Mineral Resources and the Atlantic Charter, arranged by the Division for the Social and International Relations of Science of the British Association, on July 24. 